\title{
Dai-Kou type conjugate gradient methods with a line search only using gradient
}

\section{Yuanyuan Huang* and Changhe Liu}

\section{"Correspondence:}

yyuanhuang@126.com

School of Mathematics and

Statistics, Henan University of

Science and Technology, Luoyang,

471023, P.R. China

\section{Springer}

\begin{abstract}
In this paper, the Dai-Kou type conjugate gradient methods are developed to solve the optimality condition of an unconstrained optimization, they only utilize gradient information and have broader application scope. Under suitable conditions, the developed methods are globally convergent. Numerical tests and comparisons with the PRP+ conjugate gradient method only using gradient show that the methods are efficient.
\end{abstract}

Keywords: conjugate gradient; optimality condition; line search; sufficient descent condition; global convergence

\section{Introduction}

Consider the following problem of finding $x \in R^{n}$ such that

$$
g(x)=0,
$$

where $g: R^{n} \rightarrow R^{n}$ is continuous. Throughout this paper, problem (1) corresponds to the first-order optimality condition of the unconstrained optimization

$$
\min f(x)
$$

where $f: R^{n} \rightarrow R$ is the function whose gradient is $g$.

Conjugate gradient methods are very efficient in solving large scale problem (2), if $f$ is known, due to their simple iteration and their low memory requirements. For any given starting point $x_{0} \in R^{n}$, an iterative sequence $\left\{x_{k}\right\}$ is generated by the following form:

$$
x_{k+1}=x_{k}+\alpha_{k} d_{k}
$$

where $\alpha_{k}$ is a step-length obtained by some line search, and $d_{k}$ is a search direction generated by

$$
d_{k}= \begin{cases}-g_{k}, & \text { if } k=0, \\ -g_{k}+\beta_{k} d_{k-1}, & \text { if } k \geq 1,\end{cases}
$$

where $g_{k}=g\left(x_{k}\right)$. Different choices of the parameter $\beta_{k}$ in (4) lead to different nonlinear conjugate gradient methods. The Fletcher-Reeves [1], Hestenes-Stiefel [2], Polak-Ribiére-

(c) The Author(s) 2017. This article is distributed under the terms of the Creative Commons Attribution 4.0 International License (http://creativecommons.org/licenses/by/4.0/), which permits unrestricted use, distribution, and reproduction in any medium, provided you give appropriate credit to the original author(s) and the source, provide a link to the Creative Commons license, and indicate if changes were made. 
Polyak [3, 4], Dai-Yuan [5] and Liu-Storey [6] formulas, and so on, are well-known formulas for $\beta_{k}$. Particularly, conjugate gradient methods with the following (sufficient) descent condition

$$
g_{k}^{T} d_{k} \leq-c\left\|g_{k}\right\|^{2}, \quad \forall k \geq 0, c>0,
$$

are very important and are always more efficient.

Recently, Dai and Kou [7] designed a family of conjugate gradient methods for the unconstrained nonlinear problems, the corresponding search direction is close to the direction of the scaled memoryless BFGS method. More importantly, they satisfied the sufficient descent condition (5). Numerical experiments illustrated that the Dai-Kou type conjugate gradient methods are more efficient than the Hager-Zhang type methods [8] presented by Hager and Zhang [8, 9]. For other descent conjugate gradient methods proposed by researchers, please see $[7,9-11]$ and the references therein.

For conjugate gradient methods, line search plays an important role for the global convergence. In general, the weak Wolfe line search,

$$
\begin{aligned}
& f\left(x_{k}+\alpha_{k} d_{k}\right) \leq f\left(x_{k}\right)+\delta \alpha_{k} g_{k}^{T} d_{k}, \\
& \sigma g_{k}^{T} d_{k} \leq g\left(x_{k}+\alpha_{k} d_{k}\right)^{T} d_{k},
\end{aligned}
$$

where $0<\delta<\sigma<1$, was used to obtain the step-length $\alpha_{k}$. Hager and Zhang [9] showed that the first condition (6) may never be satisfied due to the existence of the numerical errors (see also [7]). Thus, in order to avoid the numerical drawback of the weak Wolfe line search, they proposed approximate Wolfe conditions $[8,9]$, which was a combination of the weak Wolfe line search and

$$
\sigma g_{k}^{T} d_{k} \leq g\left(x_{k}+\alpha_{k} d_{k}\right)^{T} d_{k} \leq(2 \delta-1) g_{k}^{T} d_{k}
$$

where $0<\delta<1 / 2$ and $\delta<\sigma<1$. Numerical tests showed that the combined line search performed well, but there is no theory to guarantee the global convergence. Then Dai and Kou proposed an improved Wolfe line search, that is, the step-length $\alpha_{k}$ satisfied (7) and

$$
f\left(x_{k}+\alpha_{k} d_{k}\right) \leq f\left(x_{k}\right)+\min \left\{\epsilon\left|g_{k}^{T} d_{k}\right|, \delta \alpha_{k}\left|g_{k}^{T} d_{k}\right|+\eta_{k}\right\}
$$

where $0<\delta<\sigma<1, \epsilon>0$ is a constant parameter and $\left\{\eta_{k}\right\}$ is a positive sequence satisfying $\sum_{k \geq 0} \eta_{k}<+\infty$. With the improved Wolfe line search, the global convergence of Dai-Kou type conjugate gradient methods was guaranteed.

Although the Hager-Zhang type and Dai-Kou type conjugate gradient methods are efficient in solving problem (2), during the implementation of the methods, function evaluations are required. The goal of this paper is to solve problem (1) which is more general and includes some nonlinear equations, such as boundary value problems [12]. So, we hope to improve the Dai-Kou type conjugate gradient methods to directly solve problem (1) and retain their high numerical efficiency. More recently, Dong [13] embedded an Armijo-type line search only using gradient into the PRP+ conjugate gradient method [14] to solve 
problem (1), the step-length $\alpha_{k}$ satisfied

$$
g\left(x_{k}+\alpha_{k} d_{k}\right)^{T} d_{k}+\frac{1}{2} \max \left\{-\mu_{k}, 0\right\} \alpha_{k}\left\|d_{k}\right\|^{2} \leq \sigma g_{k}^{T} d_{k},
$$

where $\mu_{k}$ is a determined real number and $0<\sigma<1$. The line search allowed small choices of $\alpha_{k}$. In order to avoid this drawback, Dong [15] considered the following line search:

$$
\sigma g_{k}^{T} d_{k} \leq g\left(x_{k}+\alpha_{k} d_{k}\right)^{T} d_{k} \leq \delta g_{k}^{T} d_{k}
$$

where $0<\delta<\sigma<1$. Motivated by the work of [15], we embed the line search (11) into the Dai-Kou type conjugate gradient methods, then the improved methods of this paper have several advantages. They have the positive features of the Dai-Kou type methods for problem (2), they can be used to solve the nonlinear optimization (2) only requiring gradient information, and they can be used to solve some systems of nonlinear equations, such as those arising in boundary value problems and others.

The rest of this paper is organized as follows. In the next section, we simply review the Dai-Kou type conjugate gradient methods for unconstrained minimization and develop them to solve problem (1). In Section 3, we prove the global convergence of the improved methods under some suitable conditions. In Section 4, we select two classes of test problems to test the improved methods. One class is composed of test problems from the CUTEst test environment, and the other class is composed of some boundary value problems. The numerical performance is used to confirm their broader application and to compare with that of the PRP+ conjugate gradient method in [13]. Finally, some conclusions are given in Section 5.

\section{Algorithm}

In this section, we describe the details of the proposed methods. First, we briefly review the Dai-Kou type conjugate gradient methods in the setting of unconstrained minimization (2). We have mentioned above that nonlinear conjugate gradient methods are identified by the definitions of the parameter $\beta_{k}$ in (4). For the family of Dai-Kou type conjugate gradient methods, the parameter $\beta_{k}$ is defined as

$$
\beta_{k}^{N}\left(\tau_{k-1}\right)=\max \left\{\beta_{k}\left(\tau_{k-1}\right), \eta \frac{g_{k}^{T} d_{k-1}}{\left\|d_{k-1}\right\|^{2}}\right\}
$$

Here,

$$
\beta_{k}\left(\tau_{k-1}\right)=\frac{g_{k}^{T} y_{k-1}}{d_{k-1}^{T} y_{k-1}}-\left(\tau_{k-1}+\frac{\left\|y_{k-1}\right\|^{2}}{s_{k-1}^{T} y_{k-1}}-\frac{s_{k-1}^{T} y_{k-1}}{\left\|s_{k-1}\right\|^{2}}\right) \frac{g_{k}^{T} s_{k-1}}{d_{k-1}^{T} y_{k-1}},
$$

where $y_{k-1}=g_{k}-g_{k-1}, s_{k-1}=\alpha_{k-1} d_{k-1}=x_{k}-x_{k-1}, \tau_{k-1}$ is a parameter corresponding to the scaling parameter in the scaled memoryless BFGS method, and $\eta \in[0,1)$. The parameters $\beta_{k}$ in the Dai-Liao type methods [16] and the Hager-Zhang type methods [9] are special cases of formula (13). If $\tau_{k-1}$ is specially defined as

$$
\tau_{k-1}=\lambda \tau_{k-1}^{A}+(1-\lambda) \tau_{k-1}^{B}
$$


with $\lambda \in[0,1]$ and

$$
\begin{gathered}
\tau_{k-1}^{A}=\frac{\left\|y_{k-1}\right\|^{2}}{s_{k-1}^{T} y_{k-1}}, \\
\tau_{k-1}^{B}=\frac{s_{k-1}^{T} y_{k-1}}{\left\|s_{k-1}\right\|^{2}},
\end{gathered}
$$

then the Dai-Kou type conjugate gradient methods satisfy the sufficient descent condition (5).

The Dai-Kou type methods are very efficient in solving the unconstrained minimization, so we hope they can be used to solve problem (1) only requiring gradient information. Now we describe the improved methods in detail.

\section{Algorithm 2.1}

Step 0. Choose $x_{0} \in R^{n}$, constants $\sigma \in(0,1), \delta \in(0, \sigma), \lambda \in[0,1], \eta \in[0,1), \varepsilon>0$. Set $g_{0}:=$ $g\left(x_{0}\right)$ and $k:=0$.

Step 1. If $\left\|g_{k}\right\|_{\infty} \leq \varepsilon$, then stop.

Step 2. Generate the search direction $d_{k}$ by (4) with $\beta_{k}$ from (12), where $\tau_{k-1}$ is defined by (14).

Step 3. Find $\alpha_{k}$ such that condition (11) holds, then compute the new iterate $x_{k+1}=x_{k}+$ $\alpha_{k} d_{k}$. Set $k:=k+1$ and go to Step 1 .

In Step 3, the step-length $\alpha_{k}$ is determined following the inexact line search strategies of Algorithm 2.6 in [17]. Detailed steps are described in the following line search algorithm.

\section{Algorithm 2.2}

Step 0 . Set $u=0$ and $v=+\infty$. Choose $\alpha>0$. Set $j:=0$.

Step 1. If $\alpha$ does not satisfy

$$
g\left(x_{k}+\alpha d_{k}\right)^{T} d_{k} \leq \delta g_{k}^{T} d_{k}
$$

then set $\mathrm{j}:=\mathrm{j}+1$, and go to Step 2 . If $\alpha$ does not satisfy

$$
\sigma g_{k}^{T} d_{k} \leq g\left(x_{k}+\alpha d_{k}\right)^{T} d_{k}
$$

then set $j:=j+1$, and go to Step 3 . Otherwise, set $\alpha_{k}:=\alpha$, and return.

Step 2. Set $v=\alpha, \alpha=(u+v) / 2$. Then go to Step 1 .

Step 3. Set $u=\alpha, \alpha=2 u$. Then go to Step 1 .

The choice of the initial step-length is important for a line search. For conjugate gradient methods, it is important to make an initial guess of the step-length by utilizing the current iterative information about the problem. So, in Algorithm 2.2, we choose the initial steplength $\alpha=1 /\left\|g_{0}\right\|$ if $k=0$, and $\alpha=\alpha_{k-1} g_{k-1}^{T} d_{k-1} / y_{k-1}^{T} d_{k-1}$ if $k \geq 1$. 


\section{Convergence analysis}

Assumption 1 Assume that $f: R^{n} \rightarrow R$ is bounded below, that is, $f(x)>-\infty$ for all $x \in R^{n}$, and $f$ is continuously differentiable. Its gradient $g: R^{n} \rightarrow R^{n}$ is $L$-Lipschitz continuous, that is, there exists a constant $L>0$ such that

$$
\|g(x)-g(y)\| \leq L\|x-y\|, \quad \forall x, y \in R^{n} .
$$

Assumption 1 implies that there exists a positive constant $\hat{\gamma}$ such that

$$
\|g(x)\| \leq \hat{\gamma}, \quad \forall x \in R^{n}
$$

Lemma 3.1 Assume that $g: R^{n} \rightarrow R^{n}$ satisfies Assumption 1 . If $d_{0}=-g_{0}$ and $d_{k-1}^{T} y_{k-1} \neq 0$ for all $k \geq 1$, then

$$
g_{k}^{T} d_{k} \leq-\min \left\{\frac{3}{4}, 1-\eta\right\}\left\|g_{k}\right\|^{2}
$$

Proof Since $d_{0}=-g_{0}$, we have $g_{0}^{T} d_{0}=-\left\|g_{0}\right\|^{2}$, which satisfies (19). If

$$
\beta_{k}^{N}\left(\tau_{k-1}\right)=\frac{g_{k}^{T} y_{k-1}}{d_{k-1}^{T} y_{k-1}}-\left(\tau_{k-1}+\frac{\left\|y_{k-1}\right\|^{2}}{s_{k-1}^{T} y_{k-1}}-\frac{s_{k-1}^{T} y_{k-1}}{\left\|s_{k-1}\right\|^{2}}\right) \frac{g_{k}^{T} s_{k-1}}{d_{k-1}^{T} y_{k-1}},
$$

from Lemma 2.3 in [5], we have the result that

$$
g_{k}^{T} d_{k} \leq-\frac{3}{4}\left\|g_{k}\right\|^{2}
$$

And if

$$
\beta_{k}^{N}\left(\tau_{k-1}\right)=\eta \frac{g_{k}^{T} d_{k-1}}{\left\|d_{k-1}\right\|^{2}}
$$

it is easy to know that

$$
g_{k}^{T} d_{k} \leq-(1-\eta)\left\|g_{k}\right\|^{2}
$$

The proof is complete.

Lemma 3.2 Suppose that $f: R^{n} \rightarrow R$ is bounded below along the ray $\left\{x_{k}+\alpha d_{k} \mid \alpha>0\right\}$, its gradient $g$ is continuous, $d_{k}$ is a search direction at $x_{k}$, and $g_{k}^{T} d_{k}<0$. Then if $0<\delta<\sigma<1$, there exists $\alpha_{k}>0$ satisfying the line search (11).

Proof Define $\phi(\alpha)=f\left(x_{k}+\alpha d_{k}\right)$ and $\psi(\alpha)=f\left(x_{k}\right)+\alpha \delta g_{k}^{T} d_{k}$. Since $\phi(\alpha)$ is bounded below for all $\alpha>0,0<\delta<1$ and $g_{k}^{T} d_{k}<0$, the functions $\phi(\alpha)$ and $\psi(\alpha)$ must intersect at at least one point. Let $\alpha_{k}^{*}>0$ be the smallest intersecting value of $\alpha$, i.e.,

$$
f\left(x_{k}+\alpha_{k}^{*} d_{k}\right)=f\left(x_{k}\right)+\alpha_{k}^{*} \delta g_{k}^{T} d_{k}
$$


Since $f$ is continuously differentiable, by the mean value theorem, there exists $\alpha_{k} \in\left(0, \alpha_{k}^{*}\right)$ such that

$$
f\left(x_{k}+\alpha_{k}^{*} d_{k}\right)-f\left(x_{k}\right)=\alpha_{k}^{*} g\left(x_{k}+\alpha_{k} d_{k}\right)^{T} d_{k}
$$

By combining (20) and (21), we obtain

$$
\delta g_{k}^{T} d_{k}=g\left(x_{k}+\alpha_{k} d_{k}\right)^{T} d_{k}
$$

Furthermore,

$$
\sigma g_{k}^{T} d_{k} \leq g\left(x_{k}+\alpha_{k} d_{k}\right)^{T} d_{k}=\delta g_{k}^{T} d_{k}
$$

since $0<\delta<\sigma<1$ and $g_{k}^{T} d_{k}<0$.

Lemma 3.3 Assume that $g: R^{n} \rightarrow R^{n}$ is monotone on the interval $\left\{x_{k}+\alpha d_{k}: 0 \leq \alpha \leq \alpha_{k}\right\}$, where $\alpha_{k}$ satisfies the line search (11), then the following inequality holds:

$$
f\left(x_{k}+\alpha_{k} d_{k}\right) \leq f\left(x_{k}\right)+\delta \alpha_{k} g_{k}^{T} d_{k}
$$

where $f: R^{n} \rightarrow R$ is the function whose gradient is $g$.

Proof Since $g$ is monotone on the interval $\left\{x_{k}+\alpha d_{k}: 0 \leq \alpha \leq \alpha_{k}\right\}$, then

$$
\left(g\left(x_{k}+\alpha_{k} d_{k}\right)-g\left(x_{k}+\alpha d_{k}\right)\right)^{T}\left(\left(x_{k}+\alpha_{k} d_{k}\right)-\left(x_{k}+\alpha d_{k}\right)\right) \geq 0 .
$$

Since $\alpha \leq \alpha_{k}$, it is not difficult to get that

$$
g\left(x_{k}+\alpha d_{k}\right)^{T} d_{k} \leq g\left(x_{k}+\alpha_{k} d_{k}\right)^{T} d_{k} \leq \delta g_{k}^{T} d_{k}
$$

Applying this inequality to the following relation

$$
f\left(x_{k}+\alpha_{k} d_{k}\right)=f\left(x_{k}\right)+\int_{0}^{\alpha_{k}} g\left(x_{k}+\alpha d_{k}\right)^{T} d_{k} d \alpha
$$

yields inequality (24).

Now, we state the Zoutendijk condition [18] for the line search (11).

Lemma 3.4 Assume that $g: R^{n} \rightarrow R^{n}$ satisfies Assumption 1. Consider any iterative method in the form (3), where $d_{k}$ is a descent direction and $\alpha_{k}$ satisfies the line search (11), then

$$
\sum_{k \geq 0} \frac{\left(g_{k}^{T} d_{k}\right)^{2}}{\left\|d_{k}\right\|^{2}}<+\infty .
$$


Proof It follows from the Cauchy-Schwarz inequality, the Lipschitz condition (17) and the line search (11) that

$$
(\sigma-1) g_{k}^{T} d_{k} \leq\left(g_{k+1}-g_{k}\right)^{T} d_{k} \leq \alpha_{k} L\left\|d_{k}\right\|^{2}
$$

Then we have

$$
\alpha_{k} \geq \frac{1-\sigma}{L} \frac{-g_{k}^{T} d_{k}}{\left\|d_{k}\right\|^{2}}
$$

The formula with (24) implies that

$$
\frac{\left(g_{k}^{T} d_{k}\right)^{2}}{\left\|d_{k}\right\|^{2}} \leq \frac{L}{(1-\sigma) \delta}\left(f\left(x_{k}\right)-f\left(x_{k+1}\right)\right) \text {. }
$$

Summing (28) over $k$ and noting that $f$ is bounded below, we have that the desired result holds.

Now we discus the convergence properties of Algorithm 2.1. In the following, we will prove that if the gradient $g: R^{n} \rightarrow R^{n}$ is $\mu$-strongly monotone, that is, there exists a constant $\mu>0$ such that

$$
(g(x)-g(y))^{T}(x-y) \geq \mu\|x-y\|^{2}, \quad \forall x, y \in R^{n},
$$

Algorithm 2.1 is globally convergent with $\lim _{k \rightarrow \infty}\left\|g_{k}\right\|=0$, and for more general gradient $g: R^{n} \rightarrow R^{n}$, Algorithm 2.1 is convergent in the sense that $\liminf _{k \rightarrow \infty}\left\|g_{k}\right\|=0$.

Theorem 3.1 Assume that $g: R^{n} \rightarrow R^{n}$ satisfies Assumption 1 and is $\mu$-strongly monotone. The sequence $\left\{x_{k}\right\}$ is generated by Algorithm 2.1, then

$$
\lim _{k \rightarrow \infty}\left\|g_{k}\right\|=0
$$

Proof It follows from (17) and (29) that

$$
\begin{aligned}
& s_{k-1}^{T} y_{k-1} \leq\left\|s_{k-1}\right\|\left\|y_{k-1}\right\| \leq L\left\|s_{k-1}\right\|^{2}, \\
& \mu\left\|s_{k-1}\right\|^{2} \leq s_{k-1}^{T} y_{k-1} .
\end{aligned}
$$

By (31) and (32), it is easy to see that

$$
\begin{aligned}
& \frac{s_{k-1}^{T} y_{k-1}}{\left\|s_{k-1}\right\|^{2}} \leq L, \\
& \frac{\left\|y_{k-1}\right\|^{2}}{s_{k-1}^{T} y_{k-1}} \leq \frac{L^{2}}{\mu} .
\end{aligned}
$$

Then we have that

$$
\left|\tau_{k-1}\right| \leq(1-\lambda) \frac{L^{2}}{\mu}+\lambda L
$$


Consequently, we have that

$$
\begin{aligned}
\left|\beta_{k}\left(\tau_{k-1}\right)\right| & =\left|\frac{g_{k}^{T} y_{k-1}}{d_{k-1}^{T} y_{k-1}}-\left(\tau_{k-1}+\frac{\left\|y_{k-1}\right\|^{2}}{s_{k-1}^{T} y_{k-1}}-\frac{s_{k-1}^{T} y_{k-1}}{\left\|s_{k-1}\right\|^{2}}\right) \frac{g_{k}^{T} s_{k-1}}{d_{k-1}^{T} y_{k-1}}\right| \\
& \leq\left[\frac{(2-\lambda) L^{2}}{\mu^{2}}+\frac{(2+\lambda) L}{\mu}\right] \frac{\left\|g_{k}\right\|}{\left\|d_{k-1}\right\|} .
\end{aligned}
$$

Furthermore,

$$
\left|\beta_{k}^{N}\left(\tau_{k-1}\right)\right| \leq \max \left\{\frac{(2-\lambda) L^{2}}{\mu^{2}}+\frac{(2+\lambda) L}{\mu}, \eta\right\} \frac{\left\|g_{k}\right\|}{\left\|d_{k-1}\right\|} .
$$

Then

$$
\begin{aligned}
\left\|d_{k}\right\| & =\left\|-g_{k}+\beta_{k}^{N}\left(\tau_{k-1}\right) d_{k-1}\right\| \\
& \leq\left\|g_{k}\right\|+\left|\beta_{k}^{N}\left(\tau_{k-1}\right)\right|\left\|d_{k-1}\right\| \\
& \leq \zeta\left\|g_{k}\right\|,
\end{aligned}
$$

where $\zeta=1+\max \left\{\frac{(2-\lambda) L^{2}}{\mu^{2}}+\frac{(2+\lambda) L}{\mu}, \eta\right\}$.

By Lemmas 3.1 and 3.4, we have that

$$
\sum_{k \geq 0} \frac{\left\|g_{k}\right\|^{4}}{\left\|d_{k}\right\|^{2}}<\infty
$$

It follows from this and (35) that

$$
\sum_{k \geq 0}\left\|g_{k}\right\|^{2}<\infty
$$

which implies the desired result.

Theorem 3.2 Assume that $g: R^{n} \rightarrow R^{n}$ satisfies Assumption 1. Then Algorithm 2.1 is convergent in the sense that

$$
\liminf _{k \rightarrow \infty}\left\|g_{k}\right\|=0
$$

Proof We prove the theorem by contradiction. Assume that both $g_{k} \neq 0$ for all $k$ and $\liminf _{k \rightarrow \infty}\left\|g_{k}\right\|>0$, then there must exist some $\gamma>0$ such that

$$
\left\|g_{k}\right\| \geq \gamma, \quad \forall k \geq 0
$$

then $d_{k} \neq 0$, otherwise Lemma 3.1 would imply $g_{k}=0$.

It follows from (37), Lemma 3.1 and Lemma 3.4 that

$$
\gamma^{2} \sum_{k \geq 0} \frac{1}{\left\|d_{k}\right\|^{2}} \leq \sum_{k \geq 0} \frac{\left\|g_{k}\right\|^{2}}{\left\|d_{k}\right\|^{2}}
$$


and

$$
\sum_{k \geq 0} \frac{\left\|g_{k}\right\|^{2}}{\left\|d_{k}\right\|^{2}} \leq \sum_{k \geq 0} \frac{1}{\gamma^{2}} \frac{\left\|g_{k}\right\|^{4}}{\left\|d_{k}\right\|^{2}} \leq \frac{1}{\gamma^{2} \bar{c}} \sum_{k \geq 0} \frac{\left(g_{k}^{T} d_{k}\right)^{2}}{\left\|d_{k}\right\|^{2}}<\infty
$$

where $\bar{c}=\min \left\{\frac{3}{4}, 1-\eta\right\}$, then we have that

$$
\left\|d_{k}\right\| \rightarrow+\infty
$$

This means that there exists a positive integer $N$, for all $k \geq N$,

$$
\begin{aligned}
\beta_{k}^{N}\left(\tau_{k-1}\right) & =\beta_{k}\left(\tau_{k-1}\right) \\
& =\frac{g_{k}^{T} y_{k-1}}{d_{k-1}^{T} y_{k-1}}-\left(\tau_{k-1}+\frac{\left\|y_{k-1}\right\|^{2}}{s_{k-1}^{T} y_{k-1}}-\frac{s_{k-1}^{T} y_{k-1}}{\left\|s_{k-1}\right\|^{2}}\right) \frac{g_{k}^{T} s_{k-1}}{d_{k-1}^{T} y_{k-1}} \\
& =\frac{g_{k}^{T} y_{k-1}}{d_{k-1}^{T} y_{k-1}}-\left((1+\lambda) \frac{\left\|y_{k-1}\right\|^{2}}{s_{k-1}^{T} y_{k-1}}-\lambda \frac{s_{k-1}^{T} y_{k-1}}{\left\|s_{k-1}\right\|^{2}}\right) \frac{g_{k}^{T} s_{k-1}}{d_{k-1}^{T} y_{k-1}} .
\end{aligned}
$$

It follows from Lemma 3.1, (11) and (37) that

$$
d_{k-1}^{T} y_{k-1} \geq-(1-\sigma) g_{k-1}^{T} d_{k-1} \geq \bar{c}(1-\sigma) \gamma^{2} .
$$

It follows from (18), (33), (34), (40), (41) and the L-Lipschitz continuity of $g$ that, for all $k \geq N$,

$$
\left|\beta_{k}^{N}\left(\tau_{k-1}\right)\right| \leq \frac{\hat{\gamma}(1+\lambda)}{\bar{c}(1-\sigma) \gamma^{2}}\left(L+\frac{L^{2}}{\mu}\right)\left\|s_{k-1}\right\| .
$$

Define $u_{k}=d_{k} /\left\|d_{k}\right\|$, then similarly to the proof of Lemma 4.3 in [7], we can get the result that

$$
\left\|u_{k}-u_{k-1}\right\| \leq 2(1+\eta) \frac{\left\|g_{k}\right\|}{\left\|d_{k}\right\|}
$$

Then it follows from (38) and (43) that

$$
\sum_{k \geq 1}\left\|u_{k}-u_{k-1}\right\|^{2}<\infty
$$

From Assumption 1 and Lemma 3.3, we know that the generated sequence $\left\{x_{k}\right\}$ is bounded, then there exists some positive constant $\bar{\gamma}$ such that

$$
\left\|x_{k}\right\| \leq \bar{\gamma}, \quad \forall k \geq 0
$$

By using inequalities (42), (44) and (45), we can get the desired result similarly to the proof of items II and III of Theorem 3.2 in [9].

\section{Numerical experiments}

In this section, we did some numerical experiments to test the performance of the proposed method and compared it with the PRP + conjugate gradient method in [13]. All codes 
were written in Matlab and run on a notebook computer with an Intel(R) Core(TM) i55200U 2.20 GHz CPU, 8.00 GB of RAM and Linux operation system Ubuntu 12.04. All test problems were drawn from the CUTEst test library $[19,20]$ and the literature [12]. For the test problems from the CUTEst test library, we particularly chose the unconstrained optimization problems whose dimensions were at least 50. Different from the work in the literature such as $[5,7]$, we solved them only using gradient information. In order to confirm the broader application scope of the proposed method, some boundary value problems were selected from [12]. See Chapter 1 in [21] for the background of the boundary value problems.

In practical implementations, the stopping criterion used was $\left\|g_{k}\right\|_{\infty} \leq 10^{-3}$. For the proposed method in this paper, the values of $\sigma$ and $\delta$ in the line search (11) were taken to be 0.9 and 0.0001 , respectively, $\lambda=0.5$, and $\eta=0.5$. For the PRP + conjugate gradient, all the initial values came from the reference [13].

The numerical results are reported in Tables 1 and 2, where Name, Dim, Iter, Ng and CPU represent the name of the test problem, the dimension, the number of iterations, the number of gradient evaluations and the CPU time elapsed in seconds, respectively. '-' means the method failed to achieve the prescribed accuracy when the number of itera-

Table 1 Numerical results for test problems from the CUTEst library

\begin{tabular}{|c|c|c|}
\hline Name (Dim) & Method & Iter/Ng/CPU \\
\hline \multirow[t]{2}{*}{ ARGLINA (200) } & Dai_Kou & $14 / 28 / 1.673 \mathrm{e}-02$ \\
\hline & $\mathrm{PRP}+$ & $13 / 25 / 2.309 \mathrm{e}-02$ \\
\hline \multirow[t]{2}{*}{ ARGLINB (200) } & Dai_Kou & $22 / 43 / 2.577 \mathrm{e}-02$ \\
\hline & $\mathrm{PRP}+$ & $47 / 93 / 6.121 \mathrm{e}-02$ \\
\hline \multirow[t]{2}{*}{ ARGLINC (200) } & Dai_Kou & $22 / 43 / 2.420 \mathrm{e}-02$ \\
\hline & PRP+ & $47 / 92 / 6.144 \mathrm{e}-02$ \\
\hline \multirow[t]{2}{*}{ BDQRTIC (500) } & Dai_Kou & $118 / 264 / 3.731 \mathrm{e}-02$ \\
\hline & $\mathrm{PRP}+$ & $181 / 317 / 6.208 \mathrm{e}-02$ \\
\hline \multirow[t]{2}{*}{$\mathrm{BOX}(10,000)$} & Dai_Kou & $30 / 100 / 1.662 \mathrm{e}-01$ \\
\hline & $\mathrm{PRP}+$ & $56 / 104 / 2.615 e-01$ \\
\hline \multirow[t]{2}{*}{ BROWNAL (200) } & Dai_Kou & $22 / 42 / 1.004 \mathrm{e}-02$ \\
\hline & $\mathrm{PRP}+$ & $-/-/-$ \\
\hline \multirow[t]{2}{*}{ BROWNALE (200) } & Dai_Kou & $1 / 1 / 9.500 \mathrm{e}-05$ \\
\hline & $\mathrm{PRP}+$ & $1 / 1 / 1.070 \mathrm{e}-04$ \\
\hline \multirow[t]{2}{*}{ BRYBND $(5,000)$} & Dai_Kou & $24 / 34 / 3.827 \mathrm{e}-02$ \\
\hline & $\mathrm{PRP}+$ & $32 / 62 / 9.025 e-02$ \\
\hline \multirow[t]{2}{*}{ CHAINWOO $(4,000)$} & Dai_Kou & $223 / 361 / 2.337 \mathrm{e}-01$ \\
\hline & $\mathrm{PRP}+$ & $271 / 480 / 4.458 \mathrm{e}-01$ \\
\hline \multirow[t]{2}{*}{ CHNROSNB (50) } & Dai_Kou & $344 / 548 / 3.404 \mathrm{e}-02$ \\
\hline & PRP+ & $564 / 952 / 8.028 \mathrm{e}-02$ \\
\hline \multirow[t]{2}{*}{ CRAGGLVY $(5,000)$} & Dai_Kou & $142 / 273 / 2.638 \mathrm{e}-01$ \\
\hline & $\mathrm{PRP}+$ & $-/-/-$ \\
\hline \multirow[t]{2}{*}{ COSINE $(1,000)$} & Dai_Kou & $9 / 22 / 6.495 e-03$ \\
\hline & $\mathrm{PRP}+$ & $14 / 25 / 1.433 e-02$ \\
\hline \multirow[t]{2}{*}{ CURLY10 $(10,000)$} & Dai_Kou & $-/-/-$ \\
\hline & $\mathrm{PRP}+$ & $20,040 / 39,984 / 6.169 e+01$ \\
\hline \multirow[t]{2}{*}{ CURLY20 $(10,000)$} & Dai_Kou & $-/-/-$ \\
\hline & PRP+ & $27,216 / 54,259 / 1.278 e+02$ \\
\hline \multirow[t]{2}{*}{ DIXMAANA $(3,000)$} & Dai_Kou & $10 / 12 / 5.625 e-03$ \\
\hline & $\mathrm{PRP}+$ & $16 / 27 / 2.274 \mathrm{e}-02$ \\
\hline \multirow[t]{2}{*}{ DIXMAANB $(3,000)$} & Dai_Kou & $10 / 12 / 5.704 \mathrm{e}-03$ \\
\hline & $\mathrm{PRP}+$ & $11 / 15 / 1.145 e-02$ \\
\hline \multirow[t]{2}{*}{ DIXMAANC $(3,000)$} & Dai_Kou & $12 / 15 / 6.271 \mathrm{e}-03$ \\
\hline & PRP+ & 14/21/1.697e-02 \\
\hline \multirow[t]{2}{*}{ DIXMAAND $(3,000)$} & Dai_Kou & $14 / 17 / 1.011 \mathrm{e}-02$ \\
\hline & $\mathrm{PRP}+$ & $16 / 24 / 1.547 \mathrm{e}-02$ \\
\hline
\end{tabular}


Table 1 (Continued)

\begin{tabular}{|c|c|c|}
\hline Name (Dim) & Method & Iter/Ng/CPU \\
\hline \multirow[t]{2}{*}{ DIXMAANE $(3,000)$} & Dai_Kou & $85 / 123 / 4.520 \mathrm{e}-02$ \\
\hline & PRP+ & $80 / 152 / 8.792 \mathrm{e}-02$ \\
\hline \multirow[t]{2}{*}{ DIXMAANF $(3,000)$} & Dai_Kou & $31 / 42 / 2.522 \mathrm{e}-02$ \\
\hline & PRP+ & $30 / 41 / 4.214 \mathrm{e}-02$ \\
\hline \multirow[t]{2}{*}{ DIXMAANG $(3,000)$} & Dai_Kou & 29/40/2.873e-02 \\
\hline & $\mathrm{PRP}+$ & $27 / 35 / 2.557 \mathrm{e}-02$ \\
\hline \multirow[t]{2}{*}{ DIXMAANH $(3,000)$} & Dai_Kou & 28/37/1.468e-02 \\
\hline & PRP+ & $26 / 34 / 2.635 e-02$ \\
\hline \multirow[t]{2}{*}{ DIXMAANI $(3,000)$} & Dai_Kou & $124 / 186 / 6.319 e-02$ \\
\hline & $\mathrm{PRP}+$ & $124 / 239 / 1.124 \mathrm{e}-01$ \\
\hline \multirow[t]{2}{*}{ DIXMAANJ $(3,000)$} & Dai_Kou & $36 / 52 / 2.502 \mathrm{e}-02$ \\
\hline & $\mathrm{PRP}+$ & $31 / 43 / 3.019 e-02$ \\
\hline \multirow[t]{2}{*}{ DIXMAANK $(3,000)$} & Dai_Kou & $34 / 48 / 2.063 \mathbf{e}-02$ \\
\hline & PRP+ & $28 / 37 / 2.864 \mathrm{e}-02$ \\
\hline \multirow[t]{2}{*}{ DIXMAANL $(3,000)$} & Dai_Kou & 29/40/1.661e-02 \\
\hline & $\mathrm{PRP}+$ & $30 / 40 / 3.369 e-02$ \\
\hline \multirow[t]{2}{*}{ DIXMAANM $(3,000)$} & Dai_Kou & $104 / 154 / 6.135 \mathrm{e}-02$ \\
\hline & PRP+ & 157/305/1.407e-01 \\
\hline \multirow[t]{2}{*}{ DIXMAANN $(3,000)$} & Dai_Kou & 63/93/3.813e-02 \\
\hline & $\mathrm{PRP}+$ & $98 / 164 / 8.303 e-02$ \\
\hline \multirow[t]{2}{*}{ DIXMAANO $(3,000)$} & Dai_Kou & 59/86/2.737e-02 \\
\hline & $\mathrm{PRP}+$ & $80 / 130 / 7.730 e-02$ \\
\hline \multirow[t]{2}{*}{ DIXMAANP $(3,000)$} & Dai_Kou & $56 / 77 / 3.176 \mathrm{e}-02$ \\
\hline & $\mathrm{PRP}+$ & 72/111/6.704e-02 \\
\hline \multirow[t]{2}{*}{ DIXON3DQ $(10,000)$} & Dai_Kou & 620/945/5.557e-01 \\
\hline & $\mathrm{PRP}+$ & $1,467 / 2,933 / 2.524 \mathrm{e}+00$ \\
\hline \multirow[t]{2}{*}{ DMN15103LS (99) } & Dai_Kou & $119 / 206 / 1.417 \mathrm{e}+00$ \\
\hline & PRP+ & $39 / 106 / 1.053 e+00$ \\
\hline \multirow[t]{2}{*}{ DMN15333LS (99) } & Dai_Kou & $80 / 171 / 1.143 \mathrm{e}+00$ \\
\hline & $\mathrm{PRP}+$ & $-/-/-$ \\
\hline \multirow[t]{2}{*}{ DQDRTIC $(5,000)$} & Dai_Kou & $53 / 100 / 6.594 \mathrm{e}-02$ \\
\hline & $\mathrm{PRP}+$ & $76 / 151 / 1.327 \mathrm{e}-01$ \\
\hline DQRTIC $(5,000)$ & Dai_Kou & 18/31/1.109e-02 \\
\hline & PRP+ & $25 / 25 / 2.123 e-02$ \\
\hline EDENSCH $(1,000)$ & Dai_Kou & 28/43/1.159e-02 \\
\hline & PRP+ & $31 / 51 / 1.590 \mathbf{e}-02$ \\
\hline EG2 $(1,000)$ & Dai_Kou & 19/37/9.933e-03 \\
\hline & PRP+ & $32 / 58 / 2.803 \mathrm{e}-02$ \\
\hline EIGENALS $(2,550)$ & Dai_Kou & $24,758 / 37,853 / 2.181 \mathrm{e}+02$ \\
\hline & $\mathrm{PRP}+$ & $21,640 / 41,892 / 3.618 e+02$ \\
\hline ENGVAL1 $(1,000)$ & Dai_Kou & $25 / 35 / 6.147 \mathrm{e}-03$ \\
\hline & PRP+ & 20/28/1.253e-02 \\
\hline ERRINROS (50) & Dai_Kou & $111 / 171 / 1.860 \mathrm{e}-02$ \\
\hline & $\mathrm{PRP}+$ & $25,995 / 48,312 / 3.756 e+00$ \\
\hline ERRINRSM (50) & Dai_Kou & $419 / 805 / 4.634 e^{-02}$ \\
\hline & PRP+ & $-/-/-$ \\
\hline EXTROSNB $(1,000)$ & Dai_Kou & $652 / 1,063 / 1.300 \mathrm{e}-01$ \\
\hline & $\mathrm{PRP}+$ & $906 / 1,611 / 2.639 \mathrm{e}-01$ \\
\hline FLETBV3M $(5,000)$ & Dai_Kou & $115 / 263 / 4.331 \mathrm{e}-01$ \\
\hline & $\mathrm{PRP}+$ & $33 / 61 / 1.482 \mathrm{e}-01$ \\
\hline FLETCBV2 $(5,000)$ & Dai_Kou & $1 / 1 / 1.099 \mathrm{e}-03$ \\
\hline & $\mathrm{PRP}+$ & $1 / 1 / 1.283 \mathrm{e}-03$ \\
\hline FMINSRF2 $(5,625)$ & Dai_Kou & $251 / 386 / 2.966 \mathrm{e}-01$ \\
\hline & PRP+ & $338 / 567 / 6.821 \mathrm{e}-01$ \\
\hline FREUROTH $(5,000)$ & Dai_Kou & $191 / 331 / 2.437 \mathrm{e}-01$ \\
\hline & PRP+ & $75 / 133 / 1.523 e-01$ \\
\hline GENHUMPS $(5,000)$ & Dai_Kou & $9,378 / 20,870 / 3.155 e+01$ \\
\hline & $\mathrm{PRP}+$ & $10,235 / 17,320 / 3.504 \mathrm{e}+01$ \\
\hline GENROSE $(1,000)$ & Dai_Kou & $3,054 / 4,706 / 7.083 \mathrm{e}-01$ \\
\hline & $\mathrm{PRP}+$ & $4,947 / 8,388 / 1.792 \mathrm{e}+00$ \\
\hline HYDC20LS (99) & Dai_Kou & $2,541 / 3,952 / 4.016 \mathrm{e}-01$ \\
\hline & PRP+ & $-/-/-$ \\
\hline
\end{tabular}


Table 1 (Continued)

\begin{tabular}{|c|c|c|}
\hline Name (Dim) & Method & Iter/Ng/CPU \\
\hline \multirow[t]{2}{*}{$\operatorname{INDEF}(5,000)$} & Dai_Kou & $-/-/-$ \\
\hline & $\mathrm{PRP}+$ & $-/-/-$ \\
\hline \multirow[t]{2}{*}{ INDEFM $(1,000)$} & Dai_Kou & $-/-/-$ \\
\hline & $\mathrm{PRP}+$ & $628 / 1,271 / 5.722 \mathrm{e}-01$ \\
\hline \multirow[t]{2}{*}{ JIMACK $(3,549)$} & Dai_Kou & $716 / 1,098 / 4.231 \mathrm{e}+01$ \\
\hline & $\mathrm{PRP}+$ & $401 / 725 / 4.284 e+01$ \\
\hline \multirow[t]{2}{*}{ LIARWHD $(5,000)$} & Dai_Kou & $50 / 150 / 8.031 \mathrm{e}-02$ \\
\hline & PRP+ & $124 / 223 / 1.945 \mathrm{e}-01$ \\
\hline \multirow[t]{2}{*}{ MANCINO (100) } & Dai_Kou & 8/17/5.880e-02 \\
\hline & $\mathrm{PRP}+$ & $31 / 59 / 2.788 \mathrm{e}-01$ \\
\hline \multirow[t]{2}{*}{ MODBEALE $(10,000)$} & Dai_Kou & $371 / 738 / 1.879 \mathrm{e}+00$ \\
\hline & $\mathrm{PRP}+$ & $-/-/-$ \\
\hline \multirow[t]{2}{*}{ MOREBV $(5,000)$} & Dai_Kou & $1 / 1 / 5.170 \mathrm{e}-04$ \\
\hline & PRP+ & $1 / 1 / 7.230 \mathrm{e}-04$ \\
\hline \multirow[t]{2}{*}{ MSQRTALS $(1,024)$} & Dai_Kou & $749 / 1,148 / 1.534 \mathrm{e}+00$ \\
\hline & $\mathrm{PRP}+$ & $520 / 969 / 1.854 \mathrm{e}+00$ \\
\hline \multirow[t]{2}{*}{ MSQRTBLS $(1,024)$} & Dai_Kou & $783 / 1,196 / 1.639 \mathrm{e}+00$ \\
\hline & PRP+ & $681 / 1279 / 2.391 e+00$ \\
\hline \multirow[t]{2}{*}{$\operatorname{NCB} 20(5,010)$} & Dai_Kou & $365 / 688 / 1.466 e+00$ \\
\hline & $\mathrm{PRP}+$ & 148/248/8.941e-01 \\
\hline \multirow[t]{2}{*}{ NCB20B $(5,000)$} & Dai_Kou & $98 / 172 / 3.661 \mathrm{e}-01$ \\
\hline & $\mathrm{PRP}+$ & $77 / 131 / 4.434 \mathrm{e}-01$ \\
\hline \multirow[t]{2}{*}{ NONCVXU2 $(5,000)$} & Dai_Kou & $1,159 / 1,751 / 1.945 e+00$ \\
\hline & $\mathrm{PRP}+$ & $4,582 / 8,610 / 1.396 \mathrm{e}+01$ \\
\hline \multirow{2}{*}{ NONCVXUN $(5,000)$} & Dai_Kou & $1,247 / 1,887 / 2.110 \mathrm{e}+00$ \\
\hline & $\mathrm{PRP}+$ & $9,929 / 18,942 / 3.063 e+01$ \\
\hline \multirow[t]{2}{*}{ NONDIA $(5,000)$} & Dai_Kou & 13/23/1.189e-02 \\
\hline & PRP+ & $54 / 103 / 8.099 \mathrm{e}-02$ \\
\hline \multirow[t]{2}{*}{ NONDQUAR $(5,000)$} & Dai_Kou & $66 / 129 / 5.082 \mathrm{e}-02$ \\
\hline & $\mathrm{PRP}+$ & 139/202/1.238e-01 \\
\hline \multirow[t]{2}{*}{ OSCIGRAD $(10,000)$} & Dai_Kou & $31 / 44 / 5.616 \mathrm{e}-02$ \\
\hline & $\mathrm{PRP}+$ & $-1-1-$ \\
\hline OSCIPATH (500) & Dai_Kou & 30/78/6.678e-03 \\
\hline & $\mathrm{PRP}+$ & $-/-/-$ \\
\hline PENALTY1 $(1,000)$ & Dai_Kou & 18/28/4.520e-03 \\
\hline & PRP+ & $-/-/-$ \\
\hline PENALTY2 (200) & Dai_Kou & $112 / 164 / 2.145 \mathrm{e}-02$ \\
\hline & $\mathrm{PRP}+$ & 173/304/5.560e-02 \\
\hline PENALTY3 (200) & Dai_Kou & $-/-/-$ \\
\hline & $\mathrm{PRP}+$ & $-/-/-$ \\
\hline POWELLSG $(5,000)$ & Dai_Kou & $118 / 225 / 7.709 \mathrm{e}-02$ \\
\hline & PRP+ & $147 / 260 / 1.233 \mathrm{e}-01$ \\
\hline POWER $(10,000)$ & Dai_Kou & $22 / 25 / 1.965 \mathrm{e}-02$ \\
\hline & $\mathrm{PRP+}$ & $-/-/-$ \\
\hline QUARTC $(5,000)$ & Dai_Kou & 18/31/9.852e-03 \\
\hline & PRP+ & 25/25/2.080e-02 \\
\hline SCHMVETT $(5,000)$ & Dai_Kou & $38 / 68 / 1.145 \mathrm{e}-01$ \\
\hline & $\mathrm{PRP}+$ & $33 / 63 / 1.478 \mathrm{e}-01$ \\
\hline SENSORS (100) & Dai_Kou & $-/-/-$ \\
\hline & $\mathrm{PRP}+$ & $32 / 65 / 4.099 \mathrm{e}-01$ \\
\hline SINQUAD $(5,000)$ & Dai_Kou & $117 / 270 / 2.988 \mathrm{e}-01$ \\
\hline & $\mathrm{PRP}+$ & $182 / 342 / 5.408 \mathrm{e}-01$ \\
\hline SPARSINE $(5,000)$ & Dai_Kou & $875 / 1348 / 1.708 \mathrm{e}+00$ \\
\hline & PRP+ & $-/-/-$ \\
\hline SPARSQUR $(10,000)$ & Dai_Kou & $21 / 22 / 4.845 e-02$ \\
\hline & PRP+ & 16/16/6.262e-02 \\
\hline SPMSRTLS $(4,999)$ & Dai_Kou & 136/219/1.742e-01 \\
\hline & $\mathrm{PRP}+$ & $161 / 278 / 3.338 \mathrm{e}-01$ \\
\hline SROSENBR $(5,000)$ & Dai_Kou & $26 / 63 / 2.904 \mathrm{e}-02$ \\
\hline & $\mathrm{PRP}+$ & $33 / 57 / 4.532 \mathrm{e}-02$ \\
\hline SSBRYBND $(5,000)$ & Dai_Kou & $6,337 / 9,751 / 9.184 e+00$ \\
\hline & $\mathrm{PRP}+$ & $-/-/-$ \\
\hline
\end{tabular}


Table 1 (Continued)

\begin{tabular}{|c|c|c|}
\hline Name (Dim) & Method & Iter/Ng/CPU \\
\hline SSCOSINE $(5,000)$ & $\begin{array}{l}\text { Dai_Kou } \\
\text { PRP+ }\end{array}$ & $\begin{array}{l}-/-/ \\
-/-/-\end{array}$ \\
\hline TESTQUAD $(5,000)$ & $\begin{array}{l}\text { Dai_Kou } \\
\text { PRP+ }\end{array}$ & $\begin{array}{l}5,068 / 7,734 / 1.948 \mathrm{e}+00 \\
1,624 / 3,247 / 9.661 \mathrm{e}-01\end{array}$ \\
\hline TOINTGOR (50) & $\begin{array}{l}\text { Dai_Kou } \\
\text { PRP+ }\end{array}$ & $\begin{array}{l}131 / 195 / 1.998 \mathrm{e}-02 \\
105 / 180 / 2.060 \mathrm{e}-02\end{array}$ \\
\hline TOINTGSS $(5,000)$ & $\begin{array}{l}\text { Dai_Kou } \\
\text { PRP+ }\end{array}$ & $\begin{array}{l}18 / 37 / 2.997 \mathrm{e}-02 \\
14 / 27 / 2.830 \mathrm{e}-02\end{array}$ \\
\hline TOINTPSP (50) & $\begin{array}{l}\text { Dai_Kou } \\
\text { PRP+ }\end{array}$ & $\begin{array}{l}142 / 268 / 2.158 \mathrm{e}-02 \\
115 / 194 / 2.190 \mathrm{e}-02\end{array}$ \\
\hline TOINTQOR (50) & $\begin{array}{l}\text { Dai_Kou } \\
\text { PRP+ }\end{array}$ & $\begin{array}{l}43 / 64 / 7.463 \mathrm{e}-03 \\
41 / 81 / 9.627 \mathrm{e}-03\end{array}$ \\
\hline TQUARTIC $(5,000)$ & $\begin{array}{l}\text { Dai_Kou } \\
\text { PRP+ }\end{array}$ & $\begin{array}{l}35 / 103 / 4.848 \mathrm{e}-02 \\
68 / 120 / 7.646 \mathrm{e}-02\end{array}$ \\
\hline TRIDIA $(5,000)$ & $\begin{array}{l}\text { Dai_Kou } \\
\text { PRP+ }\end{array}$ & $\begin{array}{l}1,633 / 2,491 / 7.701 \mathrm{e}-01 \\
628 / 1,255 / 5.693 \mathrm{e}-01\end{array}$ \\
\hline VARDIM (200) & $\begin{array}{l}\text { Dai_Kou } \\
\text { PRP+ }\end{array}$ & $\begin{array}{l}18 / 18 / 1.765 e-03 \\
-/-/\end{array}$ \\
\hline VAREIGVL (50) & $\begin{array}{l}\text { Dai_Kou } \\
\text { PRP+ }\end{array}$ & $\begin{array}{l}19 / 29 / 4.227 \mathbf{e}-03 \\
23 / 39 / 6.727 \mathrm{e}-03\end{array}$ \\
\hline WOODS $(4,000)$ & $\begin{array}{l}\text { Dai_Kou } \\
\text { PRP+ }\end{array}$ & $\begin{array}{l}36 / 67 / 3.083 \mathrm{e}-02 \\
22 / 28 / 2.143 \mathrm{e}-02\end{array}$ \\
\hline
\end{tabular}

Table 2 Numerical results for some boundary value problems

\begin{tabular}{|c|c|c|}
\hline Name (Dim) & Method & Iter/Ng/CPU \\
\hline Function2 $(10,000)$ & $\begin{array}{l}\text { Dai_Kou } \\
\text { PRP+ }\end{array}$ & $\begin{array}{l}12 / 27 / 1.266 \mathrm{e}-02 \\
12 / 23 / 1.529 \mathrm{e}-02\end{array}$ \\
\hline Function6 $(10,000)$ & $\begin{array}{l}\text { Dai_Kou } \\
\text { PRP+ }\end{array}$ & $\begin{array}{l}1 / 1 / 5.010 \mathrm{e}-04 \\
1 / 1 / 4.399 \mathrm{e}-04\end{array}$ \\
\hline Function8 $(10,000)$ & $\begin{array}{l}\text { Dai_Kou } \\
\text { PRP+ }\end{array}$ & $\begin{array}{l}12 / 16 / 4.678 \mathrm{e}-02 \\
10 / 17 / 7.151 \mathrm{e}-02\end{array}$ \\
\hline Function12 $(10,000)$ & $\begin{array}{l}\text { Dai_Kou } \\
\text { PRP+ }\end{array}$ & $\begin{array}{l}10 / 21 / 1.206 \mathrm{e}-02 \\
10 / 19 / 1.227 \mathrm{e}-02\end{array}$ \\
\hline Function13 $(10,000)$ & $\begin{array}{l}\text { Dai_Kou } \\
\text { PRP+ }\end{array}$ & $\begin{array}{l}222 / 330 / 2.044 \mathrm{e}-01 \\
346 / 691 / 5.704 \mathrm{e}-01\end{array}$ \\
\hline Function14 $(10,000)$ & $\begin{array}{l}\text { Dai_Kou } \\
\text { PRP+ }\end{array}$ & $\begin{array}{l}\text { 12/17/4.554e-02 } \\
9 / 11 / 4.912 \mathrm{e}-02\end{array}$ \\
\hline Function $18(10,000)$ & $\begin{array}{l}\text { Dai_Kou } \\
\text { PRP+ }\end{array}$ & $\begin{array}{l}1 / 1 / 8.588 \mathrm{e}-04 \\
1 / 1 / 7.632 \mathrm{e}-04\end{array}$ \\
\hline Function $19(10,000)$ & $\begin{array}{l}\text { Dai_Kou } \\
\text { PRP+ }\end{array}$ & $\begin{array}{l}9 / 14 / 1.084 \mathrm{e}-02 \\
8 / 12 / 1.551 \mathrm{e}-02\end{array}$ \\
\hline Function20 $(10,000)$ & $\begin{array}{l}\text { Dai_Kou } \\
\text { PRP+ }\end{array}$ & $\begin{array}{l}1 / 1 / 7.464 \mathrm{e}-04 \\
1 / 1 / 9.391 \mathrm{e}-04\end{array}$ \\
\hline Function21 $(10,000)$ & $\begin{array}{l}\text { Dai_Kou } \\
\text { PRP+ }\end{array}$ & $\begin{array}{l}\text { 75/81/5.441e-02 } \\
-/-/-\end{array}$ \\
\hline Function22 $(10,000)$ & $\begin{array}{l}\text { Dai_Kou } \\
\text { PRP+ }\end{array}$ & $\begin{array}{l}13 / 21 / 1.300 \mathrm{e}-02 \\
12 / 21 / 1.580 \mathrm{e}-02\end{array}$ \\
\hline Function24 $(10,000)$ & $\begin{array}{l}\text { Dai_Kou } \\
\text { PRP+ }\end{array}$ & $\begin{array}{l}5 / 7 / 7.387 e+00 \\
6 / 10 / 1.609 e+01\end{array}$ \\
\hline Function25 $(10,000)$ & $\begin{array}{l}\text { Dai_Kou } \\
\text { PRP+ }\end{array}$ & $\begin{array}{l}12 / 22 / 2.008 \mathrm{e}-02 \\
16 / 26 / 4.658 \mathrm{e}-02\end{array}$ \\
\hline Function26 $(10,000)$ & $\begin{array}{l}\text { Dai_Kou } \\
\text { PRP+ }\end{array}$ & $\begin{array}{l}258 / 387 / 1.890 \mathrm{e}-01 \\
345 / 689 / 4.391 \mathrm{e}-01\end{array}$ \\
\hline Function27 $(10,000)$ & $\begin{array}{l}\text { Dai_Kou } \\
\text { PRP+ }\end{array}$ & $\begin{array}{l}143 / 212 / 1.285 e-01 \\
171 / 341 / 2.837 e-01\end{array}$ \\
\hline Function29 $(10,000)$ & $\begin{array}{l}\text { Dai_Kou } \\
\text { PRP+ }\end{array}$ & $\begin{array}{l}2,211 / 3,355 / 6.638 \mathrm{e}+00 \\
8,150 / 16,299 / 4.633 \mathrm{e}+01\end{array}$ \\
\hline Function31 $(10,000)$ & $\begin{array}{l}\text { Dai_Kou } \\
\text { PRP+ }\end{array}$ & $\begin{array}{l}1 / 1 / 5.388 \mathrm{e}-04 \\
1 / 1 / 9.083 \mathrm{e}-04\end{array}$ \\
\hline
\end{tabular}


Figure 1 Performance profile for the test problems from the CUTEst library based on the number of iterations.

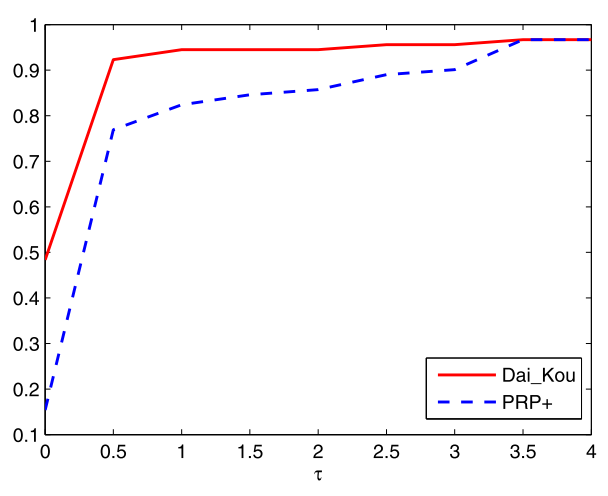

Figure 2 Performance profile for the test problems from the CUTEst library based on the number of gradient evaluations.

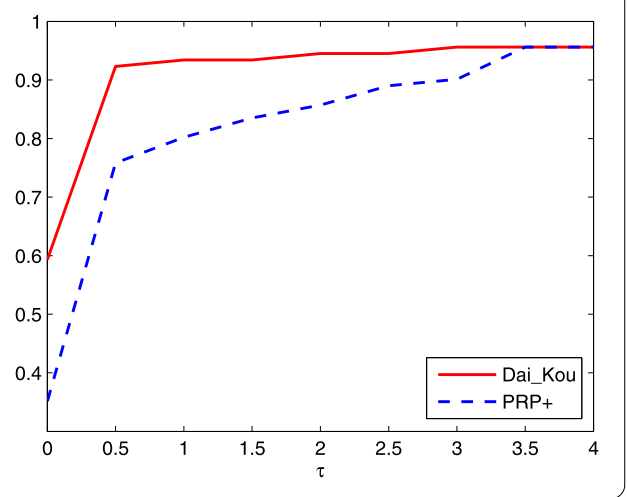

Figure 3 Performance profile for the test problems from the CUTEst library based on the CPU time.

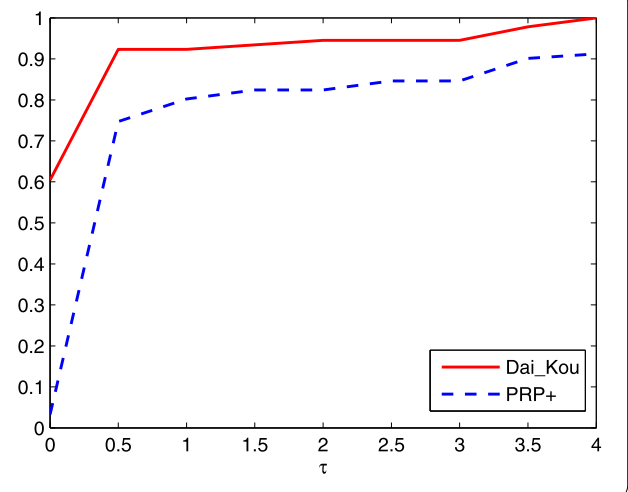

Figure 4 Performance profile for some boundary value problems based on the number of iterations.

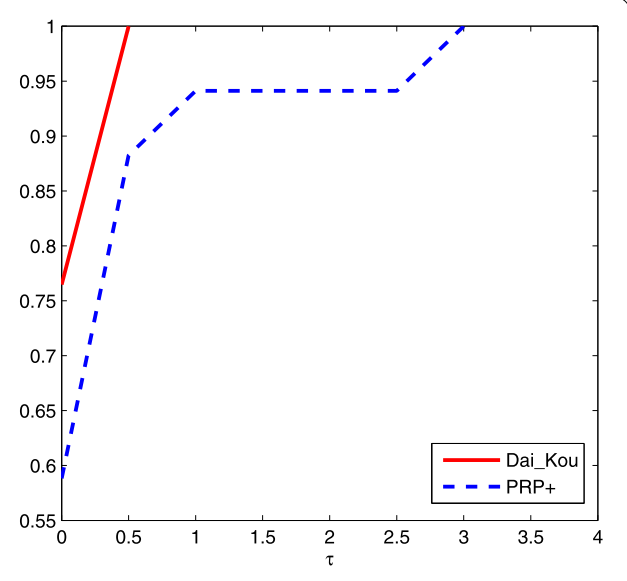



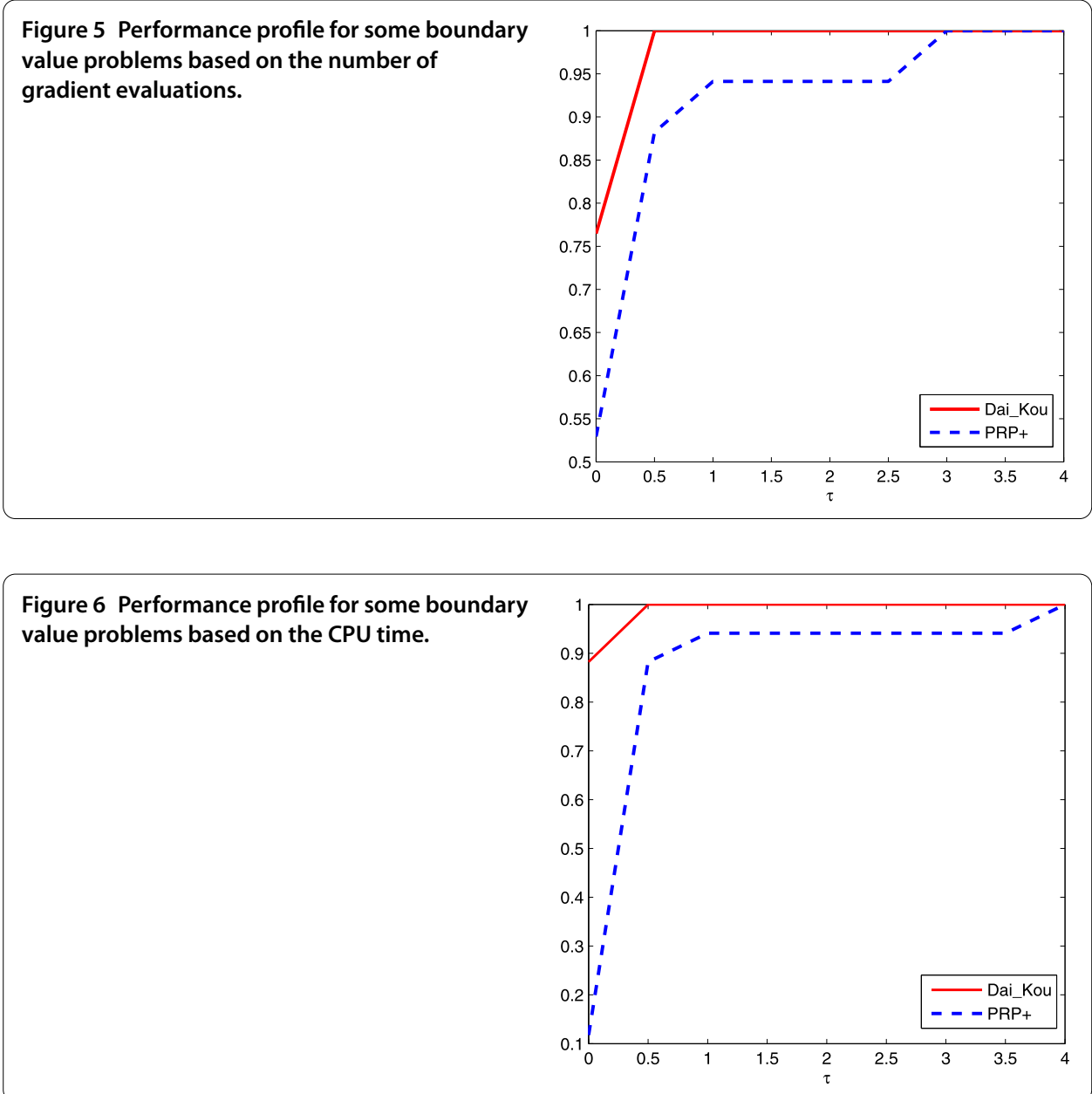

tions exceeded 50,000 or the gradient function generated 'NaN'. The performances of the two methods were evaluated using the profiles of Dolan and Morè [22]. That is, we plotted the fraction $P$ of the test problems for which each of the two methods was within a factor $\tau$. In the performance profiles, the top curve represents the most robust one within the same factor $\tau$, and the left curve represents the fastest one to solve the same percentage of test problems. Figures 1-3 show the performance profiles for test problems from the CUTEst library relating to the number of iterations, the number of gradient evaluations and the CPU time, respectively. Figures 4-6 show the performance profiles for some boundary value problems. These figures reveal that, for the test problems, the proposed method is more efficient and robust than the PRP+ conjugate gradient method. Consequently, the improved method not only can solve problems only referring to gradient information but also inherits the good numerical performance of the Dai-Kou type conjugate gradient methods.

\section{Conclusions}

In this paper, we discussed the improved Dai-Kou type conjugate gradient methods only using gradient information. They inherited the advantages of the Dai-Kou type conjugate gradient methods for solving the unconstrained minimization problems, but had broader application scope. Moreover, the problem considered in this paper can be viewed as the 
nonlinear equation

$$
F(x)=0
$$

with $F=g$. While the convergence analysis of this paper needed some assumptions of the function $f$ whose gradient is $g$, our further investigation is to avoid the function $f$ and to solve general nonlinear equation (46) using different strategies from those of this paper and literature [23-25].

\section{Competing interests}

The authors declare that they have no competing interests.

\section{Authors' contributions}

All authors read and approved the final version of this paper.

\section{Acknowledgements}

The authors are very grateful to the associate editor and reviewers for their valuable suggestions which have greatly improved the paper. This work was partially supported by the National Natural Science Foundation of China (No. 11471102) and the Key Basic Research Foundation of the Higher Education Institutions of Henan Province (No. $16 \mathrm{~A} 110012)$

\section{Publisher's Note}

Springer Nature remains neutral with regard to jurisdictional claims in published maps and institutional affiliations.

Received: 17 August 2016 Accepted: 22 March 2017 Published online: 04 April 2017

\section{References}

1. Fletcher, R, Reeves, C: Function minimization by conjugate gradients. Comput. J. 7, 149-154 (1964)

2. Hestenes, MR, Stiefel, EL: Methods of conjugate gradients for solving linear systems. J. Res. Natl. Bur. Stand. 49(5), 409-436 (1952)

3. Polak, E, Ribière, G: Note sur la méthodes de directions conjuguées. Rev. Fr. Inform. Rech. Opér., Sér Rouge 16, 35-43 (1969)

4. Polyak, BT: The conjugate gradient methods in extreme problems. USSR Comput. Math. Math. Phys. 9, 94-112 (1969)

5. Dai, YH, Yuan, Y: A nonlinear conjugate gradient method with a strong global convergence property. SIAM J. Optim. 10(1), 177-182 (1999)

6. Liu, Y, Storey, C: Efficient generalized conjugate gradient algorithms. Part 1: theory. J. Optim. Theory Appl. 69, 129-137 (1991)

7. Dai, YH, Kou, CX: A nonlinear conjugate gradient algorithm with an optimal property and an improved Wolfe line search. SIAM J. Optim. 23, 296-320 (2013)

8. Hager, WW, Zhang, HC: Algorithm 851: CG__DESCENT, a conjugate gradient method with guaranteed descent. ACM Trans. Math. Softw. 32, 113-137 (2006)

9. Hager, WW, Zhang, HC: A new conjugate gradient method with guaranteed descent and an efficient line search. SIAM J. Optim. 16, 170-192 (2005)

10. Zhang, L, Li, JL: A new globalization technique for nonlinear conjugate gradient methods for nonconvex minimization. Appl. Math. Comput. 217, 10295-10304 (2011)

11. Nakamura, W, Narushima, Y, Yabe, H: Nonlinear conjugate gradient methods with sufficient descent properties for unconstrained optimization. J. Ind. Manag. Optim. 9, 595-619 (2013)

12. Spedicato, E, Huang, Z: Numerical experience with Newton-like methods for nonlinear algebraic systems. Computing 58, 69-89 (1997)

13. Dong, YD: A practical PR+ conjugate gradient method only using gradient. Appl. Math. Comput. 219, 2041-2052 (2012)

14. Powell, MJD: Convergence properties of algorithms for nonlinear optimization. SIAM Rev. 28, 487-500 (1986)

15. Dong, YD: The BFGS method only using gradient. Preprint. http://www.optimization-online.org/DB_FILE/2016/07/5560.pdf

16. Dai, YH, Liao, LZ: New conjugacy conditions and related nonlinear conjugate gradient methods. Appl. Math. Optim. 43, 87-101 (2001)

17. Lewis, AS, Overton, ML: Nonsmooth optimization via quasi-Newton methods. Math. Program. 141(1-2), 135-163 $(2013)$

18. Zoutendijk, G: Nonlinear programming, computational methods. In: Abadie, J (ed.) Integer and Nonlinear Programming, pp. 37-86. North-Holland, Amsterdam (1970)

19. Bongartz, I, Conn, AR, Gould, N, Toint, PL: CUTE: constrained and unconstrained testing environment. ACM Trans. Math. Softw. 21, 123-160 (1995)

20. Gould, NIM, Orban, D, Toint, PL: CUTEr and SifDec: a constrained and unconstrained testing environment, revisited. ACM Trans. Math. Softw. 29, 373-394 (2003)

21. Ortega, JM, Rheinboldt, WC: Iterative Solution of Nonlinear Equations in Several Variables. Academic Press, New York (1970) 
22. Dolan, ED, Morè, JJ: Benchmarking optimization software with performance profiles. Math. Program. 91, 201-213 (2002)

23. Solodov, MV, Svaiter, BF: A globally convergent inexact Newton method for systems of monotone equations. In: Fukushima, M, Qi, L (eds.) Reformulation: Nonsmooth, Piecewise Smooth, Semismooth and Smoothing Methods, pp. 355-369. Kluwer Academic, Dordrecht (1999)

24. Yan, QR, Peng, XZ, Li, DH: A globally convergent derivative-free method for solving large-scale nonlinear monotone equations. J. Comput. Appl. Math. 234, 649-657 (2010)

25. Li, QN, Li, DH: A class of derivative-free methods for large-scale nonlinear monotone equations. IMA J. Numer. Anal. 31, 1625-1635 (2011)

Submit your manuscript to a SpringerOpen ${ }^{\circ}$ journal and benefit from:

- Convenient online submission

Rigorous peer review

- Immediate publication on acceptance

- Open access: articles freely available online

- High visibility within the field

- Retaining the copyright to your article 